\title{
Síndrome de Burnout en Funcionarios de Prisiones
}

\section{Burnout Syndrome in Prison Officers}

\author{
Percy Leonardo Álvarez-Cabrera \\ Universidad Santo Tomás de Arica \\ Fernando Chacón-Fuertes y Esteban Sánchez-Moreno \\ Universidad Complutense de Madrid
}

\begin{abstract}
El presente estudio analiza el síndrome burnout (SBO) y el afrontamiento del estrés en una muestra de 290 funcionarios/as de prisiones del norte de Chile (Región de Arica y Parinacota). Los instrumentos utilizados fueron el Maslach Burnout Inventory (MBI-HSS), el Cuestionario de Afrontamiento del Estrés y el Cuestionario de Satisfacción Laboral S20/23. Los resultados muestran una prevalencia baja de SBO (alrededor del 6\%), la que es similar a otras investigaciones, en consonancia con la evidencia empírica disponible para otros países. A través de un análisis de varianza de un factor, los resultados también sugieren un papel relevante del sexo: las mujeres presentan mayores puntuaciones en SBO. En cuanto al tipo de estrategia de afrontamiento más utilizada, se destacan la búsqueda de apoyo social, la expresión emocional abierta, la focalización en la solución del problema y la reevaluación positiva del problema. Los resultados destacan la importancia de la dimensión de despersonalización del SBO en la muestra, constituyendo un rasgo distintivo de las y los funcionarios de prisiones.
\end{abstract}

Palabras clave: burnout, afrontamiento al estrés, prisiones, oficiales correccionales

This study analyzes burnout syndrome (SBO) and stress-coping strategies in 290 prison officers from the north of Chile (Arica and Parinacota Region). The instruments used were the Maslach Burnout Inventory (MBI-HSS), the Stress Coping Questionnaire, and the Labor Satisfaction Questionnaire S20/23. Results show a low prevalence of SBO (around 6\%), which is similar to values reported in previous studies and consistent with the empirical evidence available for other countries. A single factor analysis of variance also suggests that gender plays a relevant role, as women scored higher on the SBO. The most commonly used coping strategies were searching for social support, expressing emotions openly, focusing on solving the problem, and reevaluating the problem positively. Results highlight the importance of the depersonalization dimension of the SBO in the sample, constituting a distinctive feature of prison officers.

Keywords: burnout, coping with stress, prisons, correctional officers

La investigación psicosocial sobre el síndrome de estar quemado o síndrome de burnout (en adelante, SBO) tiene su origen en los trabajos de Maslach y Jackson (1982) y Maslach (1976), quienes utilizan el concepto para describir y dar cuenta del proceso de deterioro psicosociológico característico de aquellos grupos profesionales cuyo entorno de trabajo diario está definido por una relación directa con personas que requieren ayuda o atención especial. El SBO es definido como un proceso tridimensional en el cual se da la presencia simultánea de una baja realización personal (RP) y altos niveles de agotamiento emocional (AE) y despersonalización (DP; Maslach, 2003). El SBO no es conceptualizado como una forma particular de estrés laboral, sino más bien como una respuesta al estrés laboral crónico que ocurre con frecuencia en los profesionales de las organizaciones de servicios que trabajan en contacto directo con los usuarios de tales entidades (Gil-Monte, 2003; Gil-Monte \& Peiró, 1997). De esta forma, es de esperar que la prevalencia del SBO sea especialmente significativa en aquellas profesiones en las cuales confluyen dos características, a saber, la existencia de altos niveles potenciales de estrés laboral crónico y el desempeño de las funciones y roles laborales con personas (Greig Undurraga \& Fuentes Martínez, 2009). La labor profesional de las y los oficiales correccionales, policías de prisiones o funcionarios se ajusta patentemente a esta descripción. En primer lugar, el objeto y foco fundamental del desempeño de sus funciones está constituido por personas (en

Percy Leonardo Álvarez-Cabrera, Psicología, Facultad de Ciencias Sociales y Comunicaciones, Universidad Santo Tomás de Arica, Chile; Fernando Chacón-Fuertes, Facultad de Psicología, Universidad Complutense de Madrid, España; Esteban SánchezMoreno, Instituto Universitario de Cooperación y Desarrollo, Facultad de Trabajo Social, Universidad Complutense de Madrid, España.

La correspondencia relativa a este artículo debe ser dirigida a Percy Leonardo Álvarez-Cabrera, Psicología, Facultad de Ciencias Sociales y Comunicaciones, Universidad Santo Tomás de Arica, 18 de septiembre 1191, Arica, Chile. E-mail: percyalvarez@santotomas.cl 
este caso, las personas internas o población privada de libertad) que constituyen un elemento sin el cual no puede entenderse el contexto laboral. En segundo lugar, se trata de una profesión cuyo entorno laboral se caracteriza por su alto contenido estresante y por las múltiples dimensiones que, potencialmente, pueden convertirse en estresores laborales (Lambert, Hogan \& Allen, 2006). La relación de factores organizacionales y laborales que pueden constituirse en elementos estresantes para las y los funcionarios de prisiones incluye la insuficiencia de personal, la carga de trabajo, las exigencias de la supervisión, el trabajo por turnos (frecuentemente sujeto a modificaciones), conflicto y ambigüedad de rol, relación con la jefatura y variación constante en la composición de la población interna (véase Pizarro Poggini, 2008). La literatura previa pone de manifiesto que la peligrosidad en las cárceles es un agente estresor especialmente significativo (Castle \& Martin, 2006; Cullen, Link, Wolfe \& Frank, 1985; Hernández-Martín, Fernández-Calvo, Ramos \& Contador, 2006; Millson, 2000). Se trata de uno de los elementos que caracterizan de manera evidente la labor profesional cotidiana en este grupo ocupacional, labor que se desarrolla en un entorno de violencia física potencial. Además, cuando dicha violencia tiene lugar (en forma de ataques, motines, agresiones entre los internos, suicidios, entre otros), sus efectos psicológicos en el personal de prisiones pueden prolongarse en el tiempo, más allá del momento concreto en el cual se producen. No es exagerado señalar que los contactos con una alta intensidad y carga emocionales con las personas internas constituyen, como señalan Schaufeli y Peeters (2000), la característica distintiva del trabajo de las y los funcionarios de prisiones. La interacción con los internos es una fuente de estrés crónico, encarnado de manera sistemática en situaciones y relaciones cotidianas (Finn, 2000). En este sentido, las y los funcionarios de prisiones deben implicarse diariamente en procesos de interacción social (especialmente con los internos, pero también con compañeros de trabajo) caracterizados por la ambigüedad e incertidumbre en los límites y el riesgo de implicación emocional, lo que puede derivar en conflictos interpersonales y con la propia organización. En resumen, por tanto, los factores organizacionales, las condiciones de trabajo, la centralidad de la interacción con el personal interno y la relación entre el entorno laboral y otros entornos sociales, sitúan en alto riesgo de desgaste psicosocial al personal de prisiones (Andrews \& Bonta, 2010). Por todo ello, de manera reciente la investigación psicosociológica sobre el SBO ha considerado dicho grupo (encargados de la seguridad, oficiales correccionales, entre otros) como un colectivo profesional especialmente relevante para su estudio. Durante las últimas dos décadas un número creciente de investigaciones ha permitido acumular evidencia acerca de este colectivo profesional, especialmente en Estados Unidos y Europa (Álvarez Cabrera, Chacón Fuertes, Sánchez Moreno \& Araya Urquiola, 2016; Álvarez-Cabrera, De la Cerda-Nangarí, Tordoya-Espinosa \& Arriagada-Huanca, 2018; Barra Marchant \& Sotelo Castro, 2016; Bringas-Molleda, Fernández-Muñiz, Álvarez-Fresno, Martínez-Cordero \& Rodríguez-Díaz, 2015; Gould, Watson, Price \& Valliant, 2013; Harizanova \& Tarnovska, 2013; Hernández Viejo, 2013; Hu et al., 2015; Lambert et al., 2010; López Fernández, Sáez Rodríguez, García Aranda, López Paiz \& Hernández Saavedra, 2015; Molina Brenes \& Moreno Salas, 2012; Montoya Herrera, 2015; Roy, Novak \& Miksaj-Todoravic, 2010; Schneider, 2012; Velázquez Castro et al., 2015; Oliveira, Schneider, Bonafé, Maroco \& Campos, 2016; Wang, Liu \& Wang, 2015). En América Latina las investigaciones en torno al SBO entre funcionarios/as de prisiones son escasas (Rodríguez Amaya, 2014) y en ocasiones arrojan resultados difícilmente comparables con otros contextos, dada la variedad de instrumentos de medida utilizados. En el caso de Chile, y hasta donde conocen los autores de este artículo, no existen investigaciones previas que aborden de manera específica el estudio de la prevalencia del SBO entre funcionarios/as de prisiones y su relación con otras variables laborales, organizacionales y psicosociales. En este contexto, son necesarias investigaciones que traten de medir la prevalencia del SBO entre funcionarios/as de prisiones y que se ocupen de la relación existente entre SBO y otros procesos y variables psicosociales y organizacionales características de dicho grupo laboral. Precisamente, el objetivo del presente estudio consistió en investigar el SBO en funcionarios/as de prisiones chilenos (personal de Gendarmería) destinados en la Región de Arica y Parinacota. En concreto, se formularon dos objetivos específicos. El primero, analizar la prevalencia del SBO en sus tres dimensiones en las y los funcionarios de gendarmería que desempeñan su labor en esta región, así como su asociación con las principales variables sociodemográficas y laborales. El segundo objetivo específico consistió en determinar los niveles de afrontamiento más utilizados en la muestra. Además, se plantearon las siguientes hipótesis: (a) existe una relación directa entre el uso de estrategias de afrontamiento positivas o activas y la RP y una relación directa entre el empleo de estrategias negativas o pasivas y la DP y AE; (b) variables sociodemográficas, como el sexo, la edad, el estado civil, la antigüedad en el centro y en la profesión, afectan diferencialmente a los niveles del síndrome presentados y (c) variables organizacionales relacionadas con el desempeño del puesto, como la satisfacción laboral, afectan diferencialmente a los niveles del síndrome presentados. 


\section{Método}

\section{Diseño}

Se diseñó un estudio transversal, de carácter retrospectivo y correlacional. Análisis previos de los mismos datos han sido publicados previamente (Álvarez Cabrera et al., 2016), focalizándose en análisis descriptivos y puntos de cortes del Maslach Burnout Inventory Human Services (MBI-HSS) y otras variables de riesgos psicosociales o que afectan el bienestar en el contexto penitenciario. El presente estudio se focaliza en abordar las variables de satisfacción laboral y afrontamiento al estrés.

\section{Participantes}

La población quedó constituida por todas las y los funcionarios de prisiones (gendarmes) de la Región de Arica y Parinacota $(N=334)$. De ellos, 44 no participaron en el estudio, al encontrarse en situación de licencia médica, vacaciones o permiso especial, traslado en curso o similares. El número final de participantes, por tanto, fue una muestra de 290 sujetos. De ellos, 243 eran hombres $(83,8 \%)$ y 47 mujeres $(16,2 \%)$. En cuanto al nivel educacional, 139 sujetos (47,9\%) contaban con estudios de enseñanza media completa, 27 (9,3\%), con enseñanza técnico superior y 124 sujetos $(42,8 \%)$, con estudios de educación superior. La distribución por edad muestra que 38 sujetos (13,1\%) tenían entre 18 y 26 años, $126(43,4 \%)$, entre 27 y 35 años de edad y 126 sujetos (43,4\%), más de 36 años. Según el estado civil, 98 sujetos eran solteros (33,3\%), 137, casados (46,6\%), 14 divorciados $(4,8 \%)$ y 41 convivían de hecho (15,3\%). En cuanto a la antigüedad en el centro, 34 sujetos $(11,7 \%)$ tenían entre 6 meses y 5 años de antigüedad, 49 (16,9\%) entre 5 y 10 años y 207 sujetos $(71,4 \%)$ llevaban más de 10 años en el centro. Por último, 232 sujetos tenían hijos en el momento de realizar el estudio (80\%). El número medio de hijos fue de 1,71 $(D E=0,40)$.

\section{Instrumentos}

Maslach Burnout Inventory. Para estimar el SBO se utilizó la adaptación al español (Gil-Monte, 2005), en su versión para profesionales de servicios humanos (MBI-HSS; Maslach \& Jackson, 1986), validado en Chile (Olivares Faúndez, 2009). El cuestionario consta de 22 ítems que se distribuyen en tres escalas denominadas Agotamiento Emocional, Despersonalización y Realización Personal. En todas ellas los contenidos y la frecuencia de los sentimientos descritos en sus ítems se valoraron en una escala de 7 puntos (0: Nunca, 1: Pocas veces al año o menos, 2: Una vez al mes o menos, 3: Unas pocas veces al mes o menos, 4: Una vez a la semana, 5: Pocas veces a la semana, 6: Todos los días). Un mayor puntaje muestra un mayor SBO. La escala AE está formada por nueve ítems que describen sentimientos de estar abrumado y exhausto emocionalmente por el trabajo (p. ej., Cuando me levanto por la mañana y me enfrento a otra jornada de trabajo me siento agotado). La escala DP está formada por cinco ítems que describen una respuesta impersonal y la falta de sentimientos hacia los sujetos objeto de atención (p. ej., Siento que estoy tratando a algunos internos, como si fuesen objetos impersonales). Por último, la escala RP en el trabajo está compuesta por ocho ítems que describen sentimientos de competencia y realización exitosa en el trabajo hacia los demás (p. ej., Siento que estoy influyendo positivamente en las vidas de otras personas a través de mi trabajo). Las confiabilidades que arrojaron los análisis de consistencia interna, medida a través de alfa de Cronbach, son similares al de otras investigaciones, tanto de los autores originales (Maslach \& Jackson, 1986), la validación y adaptación al castellano de Gil-Monte y Peiró (2000), la adaptación en Chile de Olivares Faúndez (2009), la aplicación en colectivos similares al de esta investigación en Sudamérica (Rodríguez Amaya, 2014) y los resultados de esta investigación (ver Tabla 1).

Sumado a lo anterior, se comprobó la dimensionalidad del Inventario de Burnout de Maslach y Jackson a través de un análisis factorial (ver Tabla 2). 
Tabla 1

Consistencia Interna del MBI-HSS en Distintos Países

\begin{tabular}{|c|c|c|c|c|}
\hline \multirow{2}{*}{ País } & \multirow{2}{*}{ Autores } & \multicolumn{3}{|c|}{ Alfa de Cronbach } \\
\hline & & $\mathrm{AE}$ & $\mathrm{DP}$ & $\mathrm{RP}$ \\
\hline Estados Unidos & Maslach \& Jackson (1986) & 0,90 & 0,70 & 0,71 \\
\hline España & Gil-Monte \& Peiró (2000) & 0,85 & 0,58 & 0,71 \\
\hline Chile & Olivares Faúndez (2009) & 0,87 & 0,69 & 0,80 \\
\hline Colombia & Rodríguez Amaya (2014) & 0,86 & 0,70 & 0,79 \\
\hline Chile & La presente investigación & 0,83 & 0,64 & 0,74 \\
\hline
\end{tabular}

Fuentes: Maslach \& Jackson, 1986; Gil-Monte \& Peiró, 2000; Olivares Faúndez, 2009; Rodríguez Amaya, 2014; Álvarez Cabrera et al., 2016.

Tabla 2

Matriz de Factores Rotados del MBI-HSS

\begin{tabular}{|c|c|c|c|}
\hline \multirow{2}{*}{ Ítem } & \multicolumn{3}{|c|}{ Factor } \\
\hline & 1 & 2 & 3 \\
\hline MBI_3 & 0,833 & & \\
\hline MBI_8 & 0,742 & & \\
\hline MBI_2 & 0,741 & & \\
\hline MBI_1 & 0,734 & & \\
\hline MBI_14 & 0,705 & & \\
\hline MBI_13 & 0,700 & & \\
\hline MBI_11 & 0,417 & & 0,409 \\
\hline MBI_10 & 0,361 & & 0,346 \\
\hline MBI_6 & 0,339 & & \\
\hline MBI_9 & & 0,628 & \\
\hline MBI_19 & & 0,609 & \\
\hline MBI_7 & & 0,516 & \\
\hline MBI_17 & & 0,506 & \\
\hline MBI_4 & & 0,487 & \\
\hline MBI_18 & & 0,482 & \\
\hline MBI_12 & $-0,382$ & 0,471 & \\
\hline MBI_21 & & 0,435 & \\
\hline MBI_22 & & & 0,710 \\
\hline MBI_20 & 0,366 & & 0,617 \\
\hline MBI_16 & 0,346 & & 0,545 \\
\hline MBI_5 & & & 0,438 \\
\hline MBI_15 & & & 0,334 \\
\hline
\end{tabular}

Nota. Los valores ennegrecidos corresponden a los ítems que coinciden con los del MBI-HSS.

Se utilizó el método de extracción de máxima verosimilitud y rotación Varimax con Kaiser, según la propuesta de Maslach y Jackson (1986), quedando la siguiente distribución de los ítems en las dimensiones:

- Dimensión 1: ítems 1, 2, 3, 6, 8, 13 y 14; sin embargo, los ítems 16 y 20 cargan en la misma dimensión pero con menor intensidad.

- Dimensión 2: ítems 4, 7, 9, 12, 17, 18, 19 y 21.

- Dimensión 3: ítems 5, 15 y 22; sin embargo, los ítems 10 y 11 cargan en la misma dimensión pero con menor intensidad. 
Por lo tanto, la dimensionalidad indica una agrupación de los ítems muy similar a la planteada por Maslach y Jackson (1986).

Para determinar el grado de burnout (alto, medio y bajo) se utilizaron los criterios normativos de GilMonte y Peiró (2000). Estos autores, al igual que en el estudio de Maslach y Jackson (1981), emplearon una clasificación tripartita de los resultados en el MBI. Según estos criterios, el patrón de puntuaciones igual o superiores a 25 en AE y superiores o igual a 9 en DP, junto a puntuaciones igual o inferiores a 40 en RP, es indicativo de alto burnout. Por el contrario, puntuaciones altas en RP (igual o superiores a 36), bajas puntuaciones en DP (igual o menores a 4) y AE (igual o menores a 16) es indicador de bajo burnout. El resto de las combinaciones de puntuaciones que no cumplan con alguno de los tres niveles de ese patrón es indicativo de que el sujeto no padece con claridad el síndrome. Es importante mencionar que se emplearon los puntos de Gil-Monte y Peiró (2000), puesto que en Chile aún no se han desarrollo investigaciones con criterios normativos en muestras carcelarias o contextos penitenciarios y el estudio de estos autores para muestras de habla hispana son los más utilizados en la literatura.

En Chile no existe validación del MBI-HSS en funcionarios/as de prisiones; el estudio de Olivares Faúndez (2009) en personal que trabaja con personas con discapacidad es lo más cercano a ello, pues utiliza los criterios de Maslach y Jackson (1986). En Sudamérica, existe una investigación que aplica el MBI-HSS de Maslach y Jackson (1986) en 111 guardianes penitenciarios de Bucaramanga, Colombia (Rodríguez Amaya, 2014).

Cuestionario de Satisfacción Laboral S20/23 (Meliá Navarro \& Peiró, 1989). Este cuestionario puede considerarse la versión reducida más completa estructuralmente de las desarrolladas a partir del S4/82 (Meliá, Peiró \& Calatayud, 1984, Septiembre). La escala total consta de 23 ítems. Estos fueron seleccionados a partir de los 82 ítems del S4/82. En Chile no se encuentran validaciones del Cuestionario de Satisfacción Laboral. Sin embargo, existen algunas investigaciones en Chile y Sudamérica que utilizan el S20/23: uno de ellos el de Jiménez Figueroa, Jara Gutiérrez y Miranda Celis (2012), que analiza burnout, apoyo social y satisfacción laboral en docentes. Sin embargo, el cuestionario no se ha utilizado en colectivos de funcionarios/as de prisiones. Su forma de respuesta es a través de una escala tipo Likert de 7 puntos (desde 1: muy satisfecho hasta 7: muy insatisfecho), de modo que a mayor puntuación mayor es la satisfacción. Posee una consistencia interna original de 0,92 (Satisfacción General), evaluada con alfa de Cronbach, y se compone de dos partes. La primera de ellas es de tipo descriptiva. La segunda parte evalúa aquellos elementos subjetivos asociados a las percepciones que los que responden tienen respecto de diferentes aspectos de su trabajo (Cornejo, Moyano, Avendaño, Acevedo \& Jiménez, 2010). La consistencia interna y algunos ítems ilustrativos para cada dimensión son: Satisfacción con la Supervisión, con seis ítems (p. ej., Estoy satisfecho de la formación que me da la empresa; $\alpha=0,88$ ), Satisfacción con el Ambiente Físico, con cinco ítems (p. ej., La limpieza e higiene de mi lugar de trabajo es buena; $\alpha=0,86)$, Satisfacción con las Prestaciones Recibidas, con cinco ítems (p. ej., Mi salario me satisface; $\alpha=0,88$ ), Satisfacción Intrínseca del Trabajo, con cuatro ítems (p. ej., Me gusta mi trabajo; $\alpha=0,82$ ) y Satisfacción con la Participación, con tres ítems (p. ej., Estoy satisfecho del ritmo a que tengo que hacer mi tarea; $\alpha=0,74)$.

Cuestionario de Afrontamiento del Estrés (CAE) de Sandín \& Chorot (2003). Es una escala de 42 ítems desarrollada y validada por los autores mencionados. Utiliza un formato de respuesta de Likert de cinco puntos (desde nunca $=0$ a casi siempre $=4$ ). Un mayor puntaje significa un mayor nivel de afrontamiento al estrés. Este instrumento ha mostrado adecuadas propiedades psicométricas medidas a través de un coeficiente promedio de consistencia interna de Cronbach de 0,79 para las siete subescalas de las que consta: Focalización en la Solución del Problema (p. ej., Analizar las causas del problema; $\alpha=0,85$ ), Autofocalización Negativa (p. ej., No hacer nada ya que las cosas suelen ser malas; $\alpha=0,64$ ), Reevaluación Positiva (p. ej., Sacar algo positivo de la situación; $\alpha=0,71$ ), Expresión Emocional Abierta (p. ej., Descargar el mal humor con los demás; $\alpha=0,74$ ), Evitación (p. ej., Concentrarse en otras cosas; $\alpha=0,76$ ), Búsqueda de Apoyo Social (p. ej., Contar los sentimientos a familiares o amigos; $\alpha=0,92$ ) y Religión (p. ej., Pedir ayuda espiritual; $\alpha=0,85)$.

\section{Procedimiento}

El estudio fue aprobado por la Comisión de Investigación y Ética de la Dirección Nacional de Gendarmería de Chile. Para la recolección de datos, se entregó a cada persona participante el cuestionario a contestar de forma autoadministrada. La cumplimentación fue llevada a cabo en el lugar de trabajo de manera 
supervisada por un investigador (psicólogo) cualificado para tal función. Se aseguró el anonimato en las respuestas, obteniendo el consentimiento informado de todos los participantes (firma de consentimiento de voluntariedad en dicha participación, la cual no implica ninguna consecuencia negativa en su trabajo). El tiempo necesario para la aplicación del instrumento fue de 15 minutos, aproximadamente.

\section{Análisis de Datos}

Se calcularon los estadísticos descriptivos de las variables centrales del estudio y la prevalencia del SBO en las tres dimensiones del cuestionario MBI-HSS. Es preciso señalar que no existe una investigación en Chile que establezca puntos de corte en una muestra normativa. Por ello, para determinar el grado de burnout (alto, medio y bajo) se siguió la estrategia propuesta por Gil-Monte y Marucco (2008), aplicando los criterios normativos procedentes de estudios de especial relevancia en la literatura sobre SBO. En concreto, se aplicaron los criterios de clasificación de Gil-Monte y Peiró (2000), Maslach y Jackson (1986), Neira (2004), Schaufeli y Van Dierendonck (1995) y Seisdedos-Cubero (1997). Para que un individuo fuera identificado como caso potencial de SBO se consideró necesario que sus puntuaciones fueran indicativas de SBO en las tres dimensiones del síndrome de manera simultánea (Gil-Monte \& Peiró, 2000; Maslach \& Jackson, 1986; Neira, 2004; Schaufeli \& Van Dierendonck, 1995; Seisdedos-Cubero, 1997). En un segundo momento del análisis, para analizar la relación entre SBO y afrontamiento y satisfacción laboral (hipótesis 1) se calcularon los coeficientes de correlación lineal de Pearson entre las diversas escalas del MBI-HSS y las dimensiones de afrontamiento y satisfacción laboral consideradas en el estudio. Por último, en un tercer momento, se realizaron comparaciones de medias de las dimensiones del MBI-HSS según variables demográficas (hipótesis 2) y de los factores de satisfacción laboral, según la presencia y ausencia del SBO (hipótesis 3), por medio de análisis de varianza (ANOVA) de un factor. El análisis de datos se realizó usando el paquete estadístico para las ciencias sociales IBM-SPSS versión 19.0.

\section{Resultados}

La Tabla 3 ofrece los estadísticos descriptivos de las principales variables del estudio.

Tabla 3

Estadísticos Descriptivos de las Escalas del MBI-HSS

\begin{tabular}{lccccr}
\hline \multicolumn{1}{c}{ Escala } & $N$ & Mínimo & Máximo & Media & $D E$ \\
\hline Agotamiento Emocional & 290 & 0 & 54 & 22,78 & 13,53 \\
Realización Personal & 290 & 6 & 48 & 33,36 & 8,75 \\
Despersonalización & 290 & 0 & 30 & 12,13 & 6,95 \\
\hline
\end{tabular}

Fuente: Álvarez Cabrera et al., 2016.

En la descripción de los niveles de SBO según los puntos de corte propuestos por Gil-Monte y Peiró (2000), Maslach y Jackson (1986), Neira (2004), Schaufeli y Van Dierendonk (1995) y Seisdedos-Cubero (1997), todos los autores utilizan como base los puntos de corte del manual del MBI-HSS para cada una de las escalas, determinando tres grados en cada una de ellas: alto, medio y bajo (Maslach \& Jackson, 1986). La diferencia en los puntos de corte de los autores está en el tipo de muestra que utilizan en sus respectivas investigaciones, destacando la de Schaufeli y Van Dierendonk (1995), pues trabajan con muestra clínicamente diagnosticadas con estrés.

En la Tabla 4 se muestra la cantidad de participantes de la muestra que presentaban el SBO, según los grados de alto, medio y bajo en las escalas del cuestionario MBI-HSS. Para la presencia de SBO se consideró que los participantes debían cumplir el criterio de puntuar bajo en RP en el trabajo, al tiempo de puntuar alto en AE y en DP. Cualquiera otra combinación se consideraba como ausencia de SBO. 
Tabla 4

Frecuencia de Burnout (SBO) y Grado de Afectación de las Escalas del MBI-HSS Obtenidas en el Estudio, según los Puntos de Corte Propuestos por Diferentes Autores

\begin{tabular}{|c|c|c|c|c|}
\hline Criterio normativo & $\begin{array}{c}\text { Escala } \\
\text { Media; } D E\end{array}$ & Puntos de corte & $\begin{array}{l}\text { Grado de } \\
\text { afectación }\end{array}$ & $f(\%)$ \\
\hline \multirow{11}{*}{$\begin{array}{l}\text { Puntos de corte de Maslach y Jackson } \\
\text { (1986) } \\
\text { Estados Unidos (personal sanitario) } \\
(N=11067)\end{array}$} & \multirow{3}{*}{$\begin{array}{l}\text { Agotamiento Emocional } \\
\text { Media }=1,02 ; D E=0,85\end{array}$} & $<17$ & Bajo & $103(35,5)$ \\
\hline & & $17-26$ & Medio & $77(26,6)$ \\
\hline & & $\geq 27$ & Alto & $110(37,9)$ \\
\hline & \multirow{3}{*}{$\begin{array}{l}\text { Despersonalización } \\
\text { Media }=1,24 ; D E=0,85\end{array}$} & $<7$ & Bajo & $78(26,9)$ \\
\hline & & $7-12$ & Medio & $64(22,1)$ \\
\hline & & $\geq 13$ & Alto & $148(51,0)$ \\
\hline & \multirow{3}{*}{$\begin{array}{l}\text { Realización Personal } \\
\text { Media }=0,92 ; D E=0,83\end{array}$} & $\geq 39$ & Bajo & $90(31,0)$ \\
\hline & & $32-38$ & Medio & $86(29,7)$ \\
\hline & & $<32$ & Alto & $114(39,3)$ \\
\hline & \multirow{2}{*}{ SBO } & \multicolumn{2}{|l|}{ Presencia de SBO } & $18(6,2)$ \\
\hline & & \multicolumn{2}{|l|}{ Ausencia de SBO } & $272(93,8)$ \\
\hline \multirow{11}{*}{$\begin{array}{l}\text { Puntos de corte de Seisdedos-Cubero } \\
\text { (1997), España } \\
\text { (muestra multiocupacional) } \\
(N=1138)\end{array}$} & \multirow{3}{*}{$\begin{array}{l}\text { Agotamiento Emocional } \\
\text { Media }=1,13 ; D E=0,83\end{array}$} & $<15$ & Bajo & $85(29,3)$ \\
\hline & & $15-24$ & Medio & $81(27,9)$ \\
\hline & & $\geq 25$ & Alto & $124(42,8)$ \\
\hline & \multirow{3}{*}{$\begin{array}{l}\text { Despersonalización } \\
\text { Media }=1,51 ; D E=0,70\end{array}$} & $<4$ & Bajo & $35(12,1)$ \\
\hline & & $4-9$ & Medio & $71(24,5)$ \\
\hline & & $\geq 10$ & Alto & $184(63,4)$ \\
\hline & \multirow{3}{*}{$\begin{array}{l}\text { Realización Personal } \\
\text { Media }=0,85 ; D E=0,81\end{array}$} & $\geq 40$ & Bajo & $78(26,9)$ \\
\hline & & $33-39$ & Medio & $92(31,7)$ \\
\hline & & $<33$ & Alto & $120(41,4)$ \\
\hline & \multirow{2}{*}{$\mathrm{SBO}$} & Presencia de SBO & & $17(5,9)$ \\
\hline & & Ausencia de SBO & & $273(94,1)$ \\
\hline \multirow{11}{*}{$\begin{array}{l}\text { Puntos de corte de Gil-Monte y Peiró } \\
(2000), \\
\text { España } \\
\text { (muestra multiocupacional) } \\
(N=1188)\end{array}$} & \multirow{3}{*}{$\begin{array}{l}\text { Agotamiento Emocional } \\
\text { Media }=1,10 ; D E=0,86\end{array}$} & $<16$ & Bajo & $95(32,8)$ \\
\hline & & $16-24$ & Medio & $71(24,5)$ \\
\hline & & $\geq 25$ & Alto & $124(42,8)$ \\
\hline & \multirow{3}{*}{$\begin{array}{l}\text { Despersonalización } \\
\text { Media }=1,54 ; D E=0,70\end{array}$} & $<4$ & Bajo & $35(12,1)$ \\
\hline & & $4-8$ & Medio & $61(21,0)$ \\
\hline & & $\geq 9$ & Alto & $194(66,9)$ \\
\hline & \multirow{3}{*}{$\begin{array}{l}\text { Realización Personal } \\
\text { Media }=0,70 ; D E=0,86\end{array}$} & $\geq 40$ & Bajo & $78(26,9)$ \\
\hline & & $36-39$ & Medio & $48(16,6)$ \\
\hline & & $<36$ & Alto & $164(56,6)$ \\
\hline & \multirow{2}{*}{ SBO } & Presencia de SBO & & $16(5,5)$ \\
\hline & & Ausencia de SBO & & $274(94,5)$ \\
\hline \multirow{11}{*}{$\begin{array}{l}\text { Puntos de corte de Neira (2004), Argentina } \\
\text { (personal sanitario) } \\
(N=1152)\end{array}$} & \multirow{3}{*}{$\begin{array}{l}\text { Agotamiento Emocional } \\
\text { Media }=1,31 ; D E=0,81\end{array}$} & $<12$ & Bajo & $65(22,4)$ \\
\hline & & $12-21$ & Medio & $69(23,8)$ \\
\hline & & $\geq 22$ & Alto & $156(53,8)$ \\
\hline & \multirow{3}{*}{$\begin{array}{l}\text { Despersonalización } \\
\text { Media }=1,62 ; D E=0,67\end{array}$} & $<3$ & Bajo & $31(10,7)$ \\
\hline & & $3-6$ & Medio & $47(16,2)$ \\
\hline & & $\geq 7$ & Alto & $212(73,1)$ \\
\hline & \multirow{3}{*}{$\begin{array}{l}\text { Realización Personal } \\
\text { Media }=0,67 ; D E=0,84\end{array}$} & $\geq 41$ & Bajo & $71(24,5)$ \\
\hline & & $36-40$ & Medio & $55(19,0)$ \\
\hline & & $<36$ & Alto & $164(56,6)$ \\
\hline & \multirow{2}{*}{ SBO } & Presencia de SBO & & $16(5,5)$ \\
\hline & & Ausencia de SBO & & $274(94,5)$ \\
\hline
\end{tabular}


Tabla 4 (conclusión)

Frecuencia de Burnout (SBO) y Grado de Afectación de las Escalas del MBI-HSS Obtenidas en el Estudio, según los Puntos de Corte Propuestos por Diferentes Autores

\begin{tabular}{|c|c|c|c|c|}
\hline Criterio normativo & $\begin{array}{c}\text { Escala } \\
\text { Media; } D E\end{array}$ & Puntos de corte & $\begin{array}{l}\text { Grado de } \\
\text { afectación }\end{array}$ & $f(\%)$ \\
\hline \multirow{11}{*}{$\begin{array}{l}\text { Puntos de corte de Schaufeli y Van } \\
\text { Dierendonk (1995), } \\
\text { Holanda } \\
\text { (muestra multiocupacional } \\
\text { diagnosticada con estrés) } \\
(N=142)\end{array}$} & \multirow{3}{*}{$\begin{array}{l}\text { Agotamiento Emocional } \\
\text { Media }=0,67 ; D E=0,86\end{array}$} & $<26$ & Bajo & $171(59,0)$ \\
\hline & & $26-33$ & Medio & $43(14,8)$ \\
\hline & & $\geq 34$ & Alto & $76(26,2)$ \\
\hline & \multirow{3}{*}{$\begin{array}{l}\text { Despersonalización } \\
\text { Media }=1,36 ; D E=0,78\end{array}$} & $<6$ & Bajo & $56(19,3)$ \\
\hline & & $6-11$ & Medio & $73(25,2)$ \\
\hline & & $\geq 12$ & Alto & $161(55,5)$ \\
\hline & \multirow{3}{*}{$\begin{array}{l}\text { Realización Personal } \\
\text { Media }=1,54 ; D E=0,76\end{array}$} & $\geq 29$ & Bajo & $206(71,0)$ \\
\hline & & $26-28$ & Medio & $36(12,4)$ \\
\hline & & $<26$ & Alto & $48(16,6)$ \\
\hline & \multirow{2}{*}{ SBO } & Presencia de SBO & & $53(18,3)$ \\
\hline & & Ausencia de SBO & & $237(81,7)$ \\
\hline
\end{tabular}

Los resultados comparativos según los diferentes criterios indican que solo el 6,2\% de los participantes presentarían el SBO si se aplicaran los puntos de corte de Maslach y Jackson (1986); resultados algo más bajos (5,9\%) arrojan las y los funcionarios de gendarmería, aplicando los puntos de corte de Seisdedos-Cubero (1997), mientras que el porcentaje es similar a 5,5\% con los criterios de Gil-Monte y Peiró (2000) y Neira (2004). Por el contrario, el SBO resultó superior cuando se utilizaron los puntos de corte de Schaufeli y Van Dierendonck (1995), pues los autores basan sus criterios en una muestra de profesionales con problemas de estrés ocupacional, único criterio validado clínicamente para el diagnóstico del burnout, lo cual se ve reflejado en un 18,3\% de los participantes que puntuaron bajo en RP y al mismo tiempo alto en AE y en DP.

En la Tabla 5 se muestran las correlaciones entre los factores del burnout, del afrontamiento y la satisfacción laboral. Se puede observar allí que la dimensión RP ofrece un mayor un número de correlaciones significativas, todas ellas directas: Búsqueda de Apoyo Social, Expresión Emocional Abierta, Focalización en la Solución del Problema, Reevaluación Positiva, Satisfacción Intrínseca del Trabajo y Satisfacción General. En cuanto a la DP, esta se correlaciona de forma directa con el factor Reevaluación Positiva e inversamente con Religión y Satisfacción con la Participación. Por otro lado, el AE se correlaciona de forma directa con Búsqueda de Apoyo Social, Religión y Focalización en la Solución del Problema y de forma inversa con Satisfacción con la Supervisión. Así, pues, la primera hipótesis formulada ha obtenido confirmación parcial en la muestra de este estudio.

Los resultados del ANOVA de un factor respecto de las dimensiones del MBI-HSS muestran que el sexo afecta directamente al AE, $F(1,288)=4,541, p=0,034$, a la DP, $F(1,288)=6,052, p=0,014$, y a la RP, $F(1,288)=3,795, p=0,052$, siendo las mujeres quienes obtienen puntuaciones más elevadas. En cambio, las variables edad, $F(2,287)=3,215, p=0,076$, estado civil, $F(3,286)=3,120, p=0,081$, y antigüedad en el centro, $F(2,287)=3,312, p=0,087$, no afectan diferencialmente los niveles del síndrome presentados en ninguna de sus tres dimensiones. Estos resultados dan cuenta de una confirmación parcial en la muestra de este estudio de la segunda hipótesis formulada, en el sentido de que solo el sexo afectaría el SBO, siendo mayores las puntuaciones en mujeres. La literatura plantea que existe una vinculación emocional mayor en este grupo, en comparación a los hombres (Álvarez Cabrera et al., 2016).

Respecto de la percepción de satisfacción laboral y SBO (presencia o ausencia), se encontró que los sujetos que no presentan el SBO puntúan más alto en Satisfacción con la Supervisión, $\eta^{2}=0,025$, Satisfacción Intrínseca del Trabajo, $\eta^{2}=0,020$, y Satisfacción General, $\eta^{2}=0,191$. En las dimensiones de Satisfacción con el Ambiente Físico, Satisfacción con las Prestaciones Recibidas y Satisfacción con la Participación no se encontraron diferencias (ver Tabla 6). Por lo que la satisfacción laboral tiene una relación en la prevención del SBO y sus consecuencias negativas para las y los funcionarios de prisión. 
Tabla 5

Correlaciones entre las Dimensiones del MBI-HSS, Factores de Afrontamiento y Factores de Satisfacción Laboral

\begin{tabular}{lccc}
\hline $\begin{array}{c}\text { Factores de afrontamiento al estrés y } \\
\text { factores de satisfacción laboral }\end{array}$ & $\begin{array}{c}\text { Agotamiento } \\
\text { emocional }\end{array}$ & Despersonalización & $\begin{array}{c}\text { Realización } \\
\text { personal }\end{array}$ \\
\hline Búsqueda de apoyo social & $0,50^{* *}$ & 0,02 & $0,65^{* *}$ \\
Expresión emocional abierta & 0,02 & 0,01 & $0,62^{* *}$ \\
Religión & $0,84^{* *}$ & $-0,23^{* *}$ & $-0,00$ \\
Focalización en la solución del problema & $0,77^{* *}$ & $-0,04$ & $0,40^{* *}$ \\
Evitación & 0,01 & 0,08 & $-0,01$ \\
Autofocalización negativa & 0,10 & 0,09 & 0,04 \\
Reevaluación positiva & 0,08 & $0,25^{* *}$ & $0,33^{*}$ \\
Satisfacción con la supervisión & $-0,30^{*}$ & 0,01 & 0,02 \\
Satisfacción con el ambiente físico & 0,14 & $-0,02$ & 0,08 \\
Satisfacción con las prestaciones recibidas & $-0,09$ & $-0,01$ & 0,00 \\
Satisfacción intrínseca del trabajo & 0,02 & 0,08 & $0,41^{* *}$ \\
Satisfacción con la participación & 0,11 & $-0,26^{*}$ & 0,01 \\
Satisfacción general & 0,03 & 0,04 & $0,72^{* *}$ \\
\hline
\end{tabular}

Fuente: Álvarez Cabrera et al., 2016.

$* * p \leq 0,01 * p \leq 0,05$.

Tabla 6

Estadísticos Descriptivos y Resultados del ANOVA en Presencia de SBO, según las Dimensiones de la Satisfacción Laboral

\begin{tabular}{lcrrrrrr}
\hline \multirow{2}{*}{ Dimensión de la satisfacción laboral } & $\begin{array}{c}\text { Presencia } \\
\text { de SBO }\end{array}$ & $N$ & Media & $D E$ & $\begin{array}{c}\text { 95\% IC de la } \\
\text { diferencia }\end{array}$ & $F$ & $p$ \\
\cline { 2 - 8 } Satisfacción con la supervisión & No & 272 & 4,26 & 1,537 & {$[1,06,2,43]$} & 4,790 & 0,029 \\
& Sí & 18 & 3,44 & 1,464 & & & \\
Satisfacción con el ambiente físico & No & 272 & 4,04 & 1,720 & & 2,912 & 0,089 \\
& Sí & 18 & 3,33 & 1,572 & & \\
Satisfacción con las prestaciones recibidas & No & 272 & 4,06 & 1,448 & & 2,597 & 0,108 \\
& Sí & 18 & 3,50 & 1,200 & & & \\
Satisfacción intrínseca del trabajo & No & 272 & 4,46 & 1,432 & {$[1,09,2,55]$} & 3,880 & 0,050 \\
& Sí & 18 & 3,78 & 1,396 & & & \\
Satisfacción con la participación & No & 272 & 2,65 & 0,905 & & 0,008 & 0,930 \\
& Sí & 18 & 2,67 & 1,029 & & \\
Satisfacción general & No & 272 & 4,18 & 1,347 & {$[1,00,2,42]$} & 4,363 & 0,038 \\
& Sí & 18 & 3,50 & 1,043 & & & \\
\hline
\end{tabular}

Nota. Grados de libertad = 1, 288.

\section{Discusión}

Se encontraron porcentajes bajos de gendarmes afectados por SBO, los cuales concuerdan con los resultados obtenidos en otras investigaciones desarrollados en diferentes contextos, como, por ejemplo, Estados Unidos (Garland, 2004; Lambert et al., 2010), Europa (Bourbonnais, Malenfant, Vézina, Jauvin \& Brisson, 2005; Cardenal Sotomayor \& Alonso Pombar, 2005; Harizanova, 2014), Israel (Keinan \& MalachPines, 2007) o Canadá (Gould et al., 2013). Los escasos estudios realizados en el contexto latinoamericano también apuntan en la misma línea. Es el caso del trabajo realizado en Jalisco (México) por Valdez Bonilla (2009), donde un bajo porcentaje de funcionarios/as de prisiones mostraron altos niveles de DP y AE, así como bajos niveles de RP, y un 4,1\% presentaba los tres rasgos del SBO. En la misma línea, la investigación realizada por Schneider (2012) en Brasil sugiere la existencia de bajos niveles de SBO, si bien en este caso 
no se realiza una estimación de su prevalencia. En el caso de Colombia, el estudio realizado por Rodríguez Amaya (2014) utilizó el cuestionario MBI-HSS para medir SBO, facilitando la comparación.

Los resultados con respecto a DP merecen especial atención, ya que sugieren una alta relevancia de esta dimensión del síndrome, constituyendo el rasgo distintivo de los gendarmes chilenos que trabajan en la región de Arica y Parinacota. Este hallazgo estaría en concordancia con los estudios de Gould et al. (2013) en Canadá y Hernández-Martín et al. (2006) y Hernández Viejo (2013) en España. Cabría pensar que la DP constituye una forma de afrontamiento de especial relevancia en el caso específico del grupo profesional que se evalúa, ya que la cosificación de los internos ayudaría a reducir el desgaste emocional de la interacción sistemática y cotidiana con dicha población. Así lo sugiere la investigación de García García y Herrero Remuzgo (2008), en la cual se halló que aquellos funcionarios/as que mantenían un contacto constante con las personas internas en un hospital psiquiátrico penitenciario mostraron mayores niveles de DP, comparándolos con el personal que mantenía un contacto relativo. Esta importancia del contacto con los internos y, en general, con las personas bajo custodia en el caso de los gendarmes, explicaría que la DP en colectivos policiales en Chile (carabineros) no surja como un rasgo tan acusado (distintivo) en la caracterización del SBO en investigaciones anteriores (Briones, 2007). En todo caso, es necesario tener en cuenta que la evidencia previa sugiere la existencia de una notable variabilidad cultural con respecto a la centralidad de la DP en el caso de las y los funcionarios de prisiones. De esta forma, Roy et al. (2010) en su estudio comparativo en torno al SBO entre funcionarios/as de prisiones en Estados Unidos y Croacia concluyen que es precisamente la DP la dimensión que en mayor medida diferencia el desarrollo del síndrome en ambos países, siendo un proceso más frecuente en las y los funcionarios estadounidenses. A la luz de esta evidencia, parece necesario el diseño de estudios que permitan dilucidar los determinantes concretos que subyacen a la DP en este colectivo profesional y que ayuden a arrojar luz en torno a este debate presente en la literatura.

En cuanto al tipo de estrategia de afrontamiento más utilizada, se destacan la búsqueda de apoyo social, la expresión emocional abierta, la focalización en el problema y la reevaluación del problema. Se puede entender que surjan estos hallazgos, pues la problemática del SBO en trabajadores penitenciarios afecta su empleo y salud física y mental, a los equipos de trabajo, sus familias, los reclusos, la organización y la sociedad (Lambert et al., 2006). Tal como señala Kalinsky (2016), el ámbito laboral penitenciario es exigente y desgastante a causa de la atención simultánea a varios frentes de potencial conflicto: la relación con cada uno de los internos, el vínculo de los internos entre sí, la relación con la jerarquía superior y con la familia, entre otros. Por lo anterior, manifestar sus emociones y buscar apoyo social y solución a sus problemáticas es parte de la realidad actual que viven los centros de reclusión.

Continuando con lo anterior, los resultados de relaciones entre variables arrojan que la dimensión RP ofrece un mayor número de asociaciones significativas con las estrategias de afrontamiento y la satisfacción laboral, todas ellas directas. Estos resultados podrían deberse a que el factor RP se relaciona más estrechamente con sentimientos de competencia, a diferencia del $\mathrm{AE}$, que se refiere a sentimientos de fatiga y cansancio, y de la DP, que se caracteriza por actitudes negativas hacia la población penitenciaria; de ahí que aquellos profesionales que se sientan competentes se caracterizarán, además, por la puesta en marcha de estrategias de afrontamiento variadas: pasivas o activas, centradas en la emoción o en el problema, en función de la problemática a la que se pueden ver enfrentados. Sumado a lo anterior, la y el funcionario penitenciario trabaja día a día en un ambiente de alta complejidad, inherentemente estresante y que implica un desgaste físico, emocional y social, entorno que requiere poner a prueba todos sus recursos personales frente al estrés laboral y manejo de las relaciones humanas.

En cuanto a la asociación entre SBO y variables sociodemográficas y laborales, los resultados solo sugieren un papel relevante del sexo: las mujeres presentan mayores puntuaciones en SBO. Algunos estudios han descrito que, por la naturaleza arriesgada y peligrosa del trabajo en prisión, las mujeres tienen una tendencia mayor a sufrir estrés (Cullen et al., 1985; Van Voorhis, Cullen, Link \& Wolfe, 1991); sin embargo, no se encontraron diferencias respecto del resto de las variables sociodemográficas estudiadas.

Por último, las y los funcionarios de prisiones más satisfechos de formal general, es decir, con mayor satisfacción intrínseca del trabajo y con la supervisión, muestran menos burnout, lo que coincide con estudios en los que se evidencian relaciones entre insatisfacción laboral y SBO (Barría, 2003; Cantisano \& Morales Domínguez, 2005). Es importante destacar que gran parte de las investigaciones en centros penitenciarios ha mostrado niveles relativamente bajos de satisfacción laboral (Castle \& Martin, 2006; Senter, 2006; Tewksbury \& Higgins, 2006). 
En conclusión, las características de la muestra y de los instrumentos de medida utilizados en la investigación respaldan la rigurosidad y utilidad de los resultados obtenidos. Estos, además, se han mostrado consistentes con la descripción teórica y conceptual del SBO, permitiendo su discusión en el contexto de la evidencia acumulada. Todo ello, unido al hecho de que esta investigación sobre SBO en funcionarios/as de prisiones en el contexto chileno es poco estudiada, invita a pensar que los hallazgos descritos suponen una aportación de relevancia al debate en Latinoamérica. En el caso concreto de Chile, país donde se realizó el estudio, sería deseable incrementar el número de regiones en las cuales se desarrollen investigaciones centradas en el impacto del SBO en el colectivo de gendarmes, propiciando, así, la posibilidad de comparación de resultados y el avance en el análisis sistemático de este objeto de estudio. En especial, los hallazgos ponen de manifiesto la centralidad de la DP entre los gendarmes chilenos, lo que sugiere que el diseño de intervenciones en el marco de dicho colectivo se beneficiaría de un énfasis en dicha dimensión. Por el mismo motivo, sería especialmente provechoso contar con una línea de investigación centrada en el estudio de las causas que subyacen a esta dimensión del SBO y de las consecuencias de los altos niveles de DP en la salud mental y en el funcionamiento laboral, profesional y social de las y los funcionarios de prisiones, pues existen investigaciones ya publicadas utilizando la misma muestra y contexto; sin embargo, no abordan las variables de satisfacción laboral y afrontamiento al estrés, por lo que la lectura de la presente investigación genera una sustantiva relevancia de dichas variables en futuras investigación, principalmente en el afrontamiento al estrés (Álvarez Cabrera et al., 2016, 2018).

El presente estudio no está exento de limitaciones. En primer lugar, su carácter transversal dificulta el establecimiento de relaciones de causalidad entre las variables. Diseños longitudinales y cuasiexperimentales permitirían un mayor detalle en el análisis de la asociación entre variables. En segundo lugar, la medición del SBO se ha realizado a través del MBI-HSS, el cuestionario más utilizado para ese propósito. Como consecuencia, es posible realizar una discusión comparativa de los resultados obtenidos y evaluar la relevancia de las diferentes dimensiones del síndrome. No obstante, conviene no olvidar las limitaciones descritas para el MBI (Olivares Faúndez \& Gil-Monte, 2009). Especialmente relevantes son algunos problemas psicométricos (estructura factorial, ambigüedad de los factores y baja confiabilidad) y conceptuales [excesiva dependencia del modelo original de Maslach y Jackson (1986) que supone un escaso contenido cognitivo y conductual de la medición], además de los diferentes puntos de corte propuestos por la literatura, lo que genera confusión al momento de puntuar o saber qué punto de corte se ajusta mejor a una determinada muestra. Esto ha llevado a que en algunos estudios se llegue a concluir, como resalta Gil-Monte (2005), que los sujetos con puntuaciones altas en dos dimensiones, o solo en una de ellas, han desarrollado el SBO. Pero, no se debería diagnosticar que un profesional padece el SBO por el solo hecho de encontrarse agotado emocionalmente o mostrarse distante y cínico con los clientes, sin tener además alteradas las otras dos dimensiones. En tercer lugar, la variabilidad en las respuestas de las y los funcionarios u omisión de algunas preguntas, sumado a la deseabilidad social, dificultó generar resultados óptimos en los análisis de datos, principalmente al momento de generar una ecuación estructural, pues, por problemas de la base de datos, no se pudo generar un modelo empírico. En cuarto lugar, la escasez de estudios sobre funcionarios/as de prisiones o contextos carcelarios en Chile, Sudamérica y Centro América dificultó la búsqueda de sustento para respaldar las hipótesis planteadas.

\section{Referencias}

Álvarez Cabrera, P. L., Chacón Fuertes, F., Sánchez Moreno, E. \& Araya Urquiola, Y. (2016). Síndrome de burnout y estrés laboral en funcionarios de prisiones en Chile. Revista Iberoamericana de Psicología, 9(1), 63-72. Extraído de https://reviberopsicologia.ibero.edu.co/article/view/913/887

Álvarez-Cabrera, P., De la Cerda-Nangarí, M., Tordoya-Espinosa, S. \& Arriagada-Huanca, N. (2018). Depresión e ideación suicida en funcionarios de gendarmería pertenecientes a guardia armada del Complejo Penitenciario de Acha y CIP CRC. Ajayu, 16(1), 171-192. Extraído de http://www.ucb.edu.bo/publicaciones/Ajayu/v16n1/v16n1_a07.pdf

Andrews, D. A. \& Bonta, J. (2010). Rehabilitating criminal justice policy and practice. Psychology, Public Policy and Law, 16, 39-55. https://doi.org/10.1037/a0018362

Barra Marchant, J. \& Sotelo Castro, R. (2016). Sindrome de burnout: una mirada a las condiciones laborales de los funcionarios de guardia armada de Gendarmería de Chile del Centro de Detención Preventiva de Puente Alto (Tesis de Grado, Escuela de Trabajo Social, Universidad Academia de Humanismo Cristiano, Santiago, Chile). Extraído de http://bibliotecadigital.academia.cl/bitstream/handle/123456789/3911/TTRASO $\% 20484$. pdf? sequence=1\&isAllowed=y

Barría, J. (2003). Síndrome de burnout en asistentes sociales del Servicio Nacional de Menores de la Región Metropolitana de Chile. Psykhe, 12(1), 213-226. Extraído de http://www.psykhe.cl/index.php/psykhe/article/viewFile/332/312

Bourbonnais, R., Malenfant, R.,Vézina, M., Jauvin, N. \& Brisson, I. (2005). Les caractéristiques du travail et la santé des agents en services de détention [Las características del trabajo y la salud de los agentes en los servicios de detención]. Revue d'Épidémiologie et de Santé Publique, 53, 127-142. https://doi.org/10.1016/S0398-7620(05)84583-3 
Bringas-Molleda, C., Fernández-Muñiz, A., Álvarez-Fresno, E., Martínez-Cordero, A. \& Rodríguez-Díaz, F. J. (2015). Influencia del síndrome de burnout en la salud de los trabajadores penitenciarios. Revista Española de Sanidad Penitenciaria, 17(3), 67-73. https://doi.org/10.4321/S1575-06202015000300002

Briones, D. (2007). Presencia del síndrome de burnout en poblaciones policiales vulnerables de Carabineros de Chile. Ciencia \& Trabajo, 9(24), 43-50. Extraído de https://www.scribd.com/document/236869254/Presencia-de-Sindrome-de-Burnout-en-Poblaciones-Policiales

Cardenal Sotomayor, V. \& Alonso Pombar, J. (2005). Un estudio sobre la incidencia del burnout entre los trabajadores del Centro Penitenciario de Huelva. Apuntes de Psicología, 23, 151-160. Extraído de http://apuntesdepsicologia.es/index.php/revista/article/view/87/89

Castle, T. L. \& Martin, J. S. (2006). Occupational hazard: Predictors of stress among jail correctional officers. American Journal of Criminal Justice, 31, 65-80. https://doi.org/10.1007/BF02885685

Cornejo, F., Moyano, E., Avendaño, M., Acevedo, M. \& Jiménez, A. (2010). Propiedades psicométricas del cuestionario de satisfacción laboral S20-23. En E. Moyano Díaz (Ed.), Calidad de vida y psicología en el Chile del bicentenario (pp. 30-42). Santiago, Chile: Talca.

Cullen, F. T., Link, B. G., Wolfe, N. T. \& Frank, J. (1985). The social dimensions of correctional officer stress. Justice Quarterly, 2, 505-533. https://doi.org/10.1080/07418828500088711

Finn, P. (2000). Addressing correctional officer stress: Programs and strategies. Washington, DC: U.S. Department of Justice, Office of Justice Programs, National Institute of Justice.

García García, J. M. \& Herrero Remuzgo, S. (2008). Variables sociodemográficas y síndrome de burnout en una muestra multiocupacional del Hospital Psiquiátrico Penitenciario de Sevilla. Apuntes de Psicología, 26, 459-477. Extraído de http://www.apuntesdepsicologia.es/index.php/revista/article/viewFile/167/169

Garland, B. (2004). The impact of administrative support on prison treatment staff burnout: An exploratory study. The Prison Journal, 84, 452-471. https://doi.org/10.1177/0032885504269343

Gil-Monte, P. R. (2003). El síndrome de quemarse por el trabajo (síndrome de burnout) en profesionales de enfermería. Revista Electrônica InterAçâo Psy, 1, 19-33. Extraído de https://docplayer.es/16461183-Revista-eletronica-interacao-psy-ano-1-no-1-ago2003-p-19-33-19-el-sindrome-de-quemarse-por-el-trabajo-sindrome-de.html

Gil-Monte, P. R. (2005). El síndrome de quemarse por el trabajo (burnout). Una enfermedad laboral en la sociedad del bienestar. Madrid, España: Pirámide.

Gil-Monte, P. R. \& Marucco, M. A. (2008). Prevalencia del "síndrome de quemarse por el trabajo" (burnout) en pediatras de hospitales generales. Revista de Saúde Pública, 42, 450-456. https://doi.org/10.1590/S0034-89102008000300009

Gil-Monte, P. \& Peiró, J. M. (1997). Desgaste psíquico en el trabajo: el síndrome de quemarse. Madrid, España: Síntesis.

Gil-Monte, P. R. \& Peiró, J. M. (2000). Un estudio comparativo sobre criterios normativos y diferenciales para el diagnóstico del síndrome de quemarse por el trabajo (burnout) según el MBI-HSS en España. Revista de Psicología del Trabajo y de las Organizaciones, 16, 135-149. Extraído de https://www.uv.es/unipsico/pdf/Publicaciones/Articulos/01_SQT/2000_RPTO_Normas.pdf

Gould, D. D., Watson, S. L., Price, S. R. \& Valliant, P. M. (2013). The relationship between burnout and coping in adult and young offender center correctional officers: An exploratory investigation. Psychological Services, 10, 37-47. https://doi.org/10.1037/a0029655

Greig Undurraga, D. \& Fuentes Martínez, X. (2009). "Burnout" o agotamiento en la práctica diaria de la medicina. Revista Chilena de Cardiología, 28, 403-407. https://doi.org/10.4067/S0718-85602009000300013

Harizanova, S. N. (2014). Job burnout among Bulgarian prison staff. International Journal of Scientific Research, 3(8), 307-308. https://doi.org/10.15373/22778179/August2014/92

Harizanova, S. N. \& Tarnovska, T. H. (2013). Professional burnout syndrome among correctional facility officers. Folia Medica, 55(2), 73-79. https://doi.org/10.2478/folmed-2013-0020

Hernández-Martín, L., Fernández-Calvo, B., Ramos, F. \& Contador, I. (2006). El síndrome de burnout en funcionarios de vigilancia de un centro penitenciario. International Journal of Clinical and Health Psychology, 6, 599-611. Extraído de https://www.redalyc.org/pdf/337/33760306.pdf

Hernández Viejo, A. (2013). El síndrome de burnout en funcionarios del Centro Penitenciario de Zuera (Zaragoza) (Trabajo de Grado, Universidad de Zaragoza, Facultad de Ciencias Sociales y Humanas de Teruel, España). Extraído de http://zaguan.unizar.es/record/12293?ln=es

Hu, S., Wang, J. -N., Liu, L., Wu, H., Yang, X., Wang, Y. \& Wang, L. (2015). The association between work-related characteristic and job burnout among Chinese correctional officers: A cross-sectional survey. Public Health, 129, $1172-1178$. https://doi.org/10.1016/j.puhe.2015.05.006

Jiménez Figueroa, A. E., Jara Gutiérrez, M. J. \& Miranda Celis, E. R. (2012). Burnout, apoyo social y satisfacción laboral en docentes. Psicologia Escolar e Educacional, 16, 125-134. https://doi.org/10.1590/S1413-85572012000100013

Kalinsky, B. (2016). La cárcel hoy. Un estudio de caso en Argentina. Revista de Historia de las Prisiones, 3, 19-34. Extraído de http://www.revistadeprisiones.com/wp-content/uploads/2016/12/3.pdf

Keinan, G. \& Malach-Pines, A. (2007). Stress and burnout among prison personnel: Sources, outcomes, and intervention strategies. Criminal Justice and Behavior, 34, 380-398. https://doi.org/10.1177/0093854806290007

Lambert, E. G., Hogan, N. L. \& Allen, R. I. (2006). Correlates of correctional officer job stress: The impact of organizational structure. American Journal of Criminal Justice, 30, 227-246. https://doi.org/10.1007/BF02885893

Lambert, E. G., Hogan, N. L., Jiang, S., Elechi, O. O., Benjamin, B., Morris, A. ... Dupuy, P. (2010). The relationship among distributive and procedural justice and correctional life satisfaction, burnout, and turnover intent: An exploratory study. Journal of Criminal Justice, 38, 7-16. https://doi.org/10.1016/j.jcrimjus.2009.11.002

López Fernández, A., Sáez Rodríguez, D., García Aranda, M. J., López Paiz, A. \& Hernández Saavedra, L. (2015). Estudio sobre las relaciones del síndrome de burnout con algunos factores psicosociales. ReiDoCrea, 4, 94-99. Extraído de http://digibug.ugr.es/bitstream/handle/10481/37009/ReiDoCrea-Vol.4Art.13.pdf;jsessionid=72B17D52011AA5820D2D48083769E9DC?sequence=1

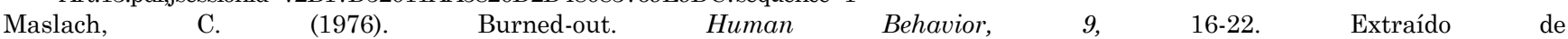
https://www.scirp.org/(S(i43dyn45teexjx455qlt3d2q))/reference/ReferencesPapers.aspx?ReferenceID=1415508

Maslach, C. (2003). Job burnout: New directions in research and intervention. Current Directions in Psychological Science, 12, $189-192$. https://doi.org/10.1111/1467-8721.01258

Maslach, C. \& Jackson, S. E. (1982). Burnout in health professions: A social psychological analysis. En G. S. Sanders \& J. Suls (Eds.), Social psychology of health and illness (pp. 227-251). Hillsdale, NJ: Lawrence Erlbaum.

Maslach, C. \& Jackson, S. E. (1986). The Maslach Burnout Inventory manual (2a ed.). Palo Alto, CA: Consulting Psychologists Press. 
Meliá, J. L., Peiró, J. M. \& Calatayud, C. (1984, Septiembre). La satisfacción de los miembros de la organización. Ponencia presentada en el I Congreso de Evaluación Psicológica, Madrid, España.

Meliá Navarro, J. L. \& Peiró, J. M. (1989). La medida de la satisfacción laboral en contextos organizacionales. El Cuestionario de Satisfacción S20/23. Psicologemas, 5, 59-74. Extraído de https://www.uv.es/meliaj1/Research/Art_Satisf/ArtS20_23.PDF

Millson, W. (2000). Predictors of work stress among correctional officers (Tesis de Magíster, Carleton University, Ottawa, Canadá). Extraído de https://www.csc-scc.gc.ca/research/forum/e141/1411_e.pdf

Montoya Herrera, P. C. (2015). Características del síndrome de burnout en funcionarios del Instituto Penitenciario de Mediana Seguridad y Carcelario de Yarumal (Trabajo de Grado, Universidad de Antioquia, Facultad de Ciencias Sociales y Humanas, Medellín, Colombia). Extraído de http://200.24.17.74:8080/jspui/bitstream/fcsh/178/1/MontoyaPaula_caracteristicassindromeburnoutfuncioanriosinstitutopenitenci ariomedianaseguridadcarcelarioyarumal.pdf

Molina Brenes, M. A. \& Moreno Salas, M. (2012). Síndrome del burnout y engagement en profesionales del sistema penitenciario costarricense. Revista de Ciencias Sociales Universidad de Costa Rica, 137, 65-81. Extraído de https://revistas.ucr.ac.cr/index.php/sociales/article/view/8419/7942

Neira, C. (2004). Cuando se enferman los que curan. Estrés laboral y burnout en los profesionales de la salud. Buenos Aires, Argentina: Gambacop.

Olivares Faúndez, V. E. (2009). Análisis de las propiedades psicométricas del Maslach Burnout Inventory Human Services (MBI-HSS) en profesionales chilenos. Ciencia \& Trabajo, 34, 217-221. Extraído de https:/www.prevencionintegral.com/canal-orp/papers/orp-2009/analisis-propiedadespsicometricas-maslach-burnout-inventory-human-services-mbi-hss-en-profesionales

Olivares Faúndez, V. E. \& Gil-Monte, P. R. (2009). Análisis de las principales fortalezas y debilidades del "Maslach Burnout Inventory" (MBI). Ciencia \& Trabajo, 33, 160-167. Extraído de https://www.researchgate.net/profile/Fernando_AriasGalicia/publication/28322448_Estres_Agotamiento_Profesional_burnout_y_Salud_en_Profesores_de_Acuerdo_a_su_Tipo_de_Contrato/links/02e7 e53190d73d46d8000000.pdf\#page=62

Oliveira, R. V., Schneider, V., Bonafé, F. S. S., Maroco, J. \& Campos J. A. D. B. (2016). Occupational characteristics and burnout syndrome in Brazilian correctional staff. Work, 55, 215-223. https://doi.org/10.3233/WOR-162367

Pizarro Poggini, A. (2008). Estrés en funcionarios penitenciarios. Revista de Estudios Criminológicos y Penitenciarios, 12, 617-632. Extraído de https://es.slideshare.net/danilosaavedra11/estres-en-funcionarios-penitenciarios

Rodríguez Amaya, R. M. (2014). Síndrome de burnout en guardianes penitenciarios, Bucaramanga, Colombia, 2013. Revista Colombiana de Psiquiatría, 43, 146-153. https://doi.org/10.1016/j.rcp.2014.03.001

Roy, S., Novak, T. \& Miksaj-Todorovic, L. (2010). Job burnout among prison staff in the United States and Croatia: A preliminary comparative study. International Journal of Criminal Justice Sciences, 5, 189-202. Extraído de http://citeseerx.ist.psu.edu/viewdoc/download?doi=10.1.1.673.8311\&rep=rep1\&type=pdf

Sandín, B. \& Chorot, P. (2003) Cuestionario de Afrontamiento del Estrés (CAE): desarrollo y validación preliminar. Revista de Psicopatología y Psicología Clínica, 8, 39-53. https://doi.org/10.5944/rppc.vol.8.num.1.2003.3941

Schaufeli, W. B. \& Peeters, M. C. W. (2000). Job stress and burnout among correctional officers: A literature review. International Journal of Stress Management, 7, 19-48. https://doi.org/10.1023/A:1009514731657

Schaufeli, W. B. \& Van Dierendonck, D. (1995). A cautionary note about the cross-national and clincal validity of cut-off points for the Maslach Burnout Inventory. Psychological Reports, 76, 1083-1090. https://doi.org/10.2466/pr0.1995.76.3c.1083

Schneider, V. C. (2012). Síndrome de burnout e o consumo de álcool em funcionários de penitenciárias do municipio de Itirapina-SP [Síndrome de burnout y el consumo de alcohol en funcionarios de penitenciarias del municipio de Itirapina-SP] (Disertación de Magíster, Universidade Estadual Paulista, Araraquara, Brasil). Extraído de https://docplayer.com.br/4981992-Sindrome-deburnout-e-o-consumo-de-alcool-em-funcionarios-de-penitenciarias-do-municipio-de-itirapina-sp.html

Seisdedos-Cubero, N. (1997). Versión española del Maslach Burnout Inventory. Madrid, España: TEA.

Senter, A. (2006). Correctional psychologist burnout, job satisfaction, and life satisfaction (Disertación Doctoral, Texas Tech University, Lubbock, TX, Estados Unidos). Extraído de http://citeseerx.ist.psu.edu/viewdoc/download?doi=10.1.1.872.6808\&rep=rep1\&type=pdf

Tewksbury, R. \& Higgins, G. E. (2006). Examining the effect of emotional dissonance on work stress and satisfaction with supervisors among correctional staff. Criminal Justice Policy Review, 17, 290-301. https://doi.org/10.1177/0887403405282961

Topa Cantisano, G. \& Morales Domínguez, J. (2005). Determinantes específicos de la satisfacción laboral, el burnout y sus consecuencias para la salud: un estudio exploratorio con funcionarios(as) de prisiones. International Journal of Psychology and Psychological Therapy, 5, 73-83. Extraído de http://www.redalyc.org/pdf/560/56050106.pdf

Valdez Bonilla, H. (2009). Detección y tratamiento del síndrome de burnout en el sistema penitenciario de Jalisco. Waxapa, 1, 24-34. Extraído de https://www.medigraphic.com/pdfs/waxapa/wax-2009/wax091e.pdf

Van Voorhis, P., Cullen, F. T., Link, B. G. \& Wolfe, N. T. (1991). The impact of race and gender on correctional officers' orientation to the integrated environment. Journal of Research in Crime and Delinquency, 28, 472-500. https://doi.org/10.1177/0022427891028004007

Velázquez Castro, T., Bracco Bruce, L., Hildenbrand Mellet, A., Wakeham Nieri, A., Valdez Oyague, R., Florentini Castañeda, M. ... Oviedo Rosas, V. (2015). Síndrome de agotamiento profesional en trabajadores de tratamiento de dos establecimientos penitenciarios de Lima. Apuntes de Psicología, 33, 57-65. Extraído de http://www.apuntesdepsicologia.es/index.php/revista/article/view/563/426

Wang, S., Liu, Y. \& Wang, L. (2015). Nurse burnout: Personal and environmental factors as predictors. International Journal of Nursing Practice, 21, 78-86. https://doi.org/10.1111/ijn.12216

Fecha de recepción: Octubre de 2017.

Fecha de aceptación: Enero de 2019. 